\title{
MedienPädagogik
}

Zeitschrift für Theorie und Praxis der Medienbildung

Themenheft Nr. 32: Offenheit in Lehre und Forschung - Königsweg oder Sackgasse? Herausgegeben von Franziska Bellinger und Anna Heudorfer

\section{Durch Studierende Offenheit in die Lehre integrieren}

\section{Das Projekt eScouts OER/MOOCs an der Ruhr-Universität Bochum}

Kathrin Braungardt, Matthias Kostrzewa und Christine Ruthenfranz

\begin{abstract}
Zusammenfassung
Open Educational Resources (OER) sind in den letzten Jahren im deutschsprachigen Raum verstärkt in den Blick der Hochschulbildung geraten. In diesem Zuge wird auch angestrebt Offenheit durch frei lizenzierte Bildungsmaterialien zu fördern und in die Breite der universitären Lehre zu bringen. Studierende können in diesem Prozess entscheidende Multiplikatorinnen und Multiplikatoren sein und als Unterstützung von Lehrenden fungieren. Wie Studierende das Thema Open Educational Resources in die Lehre ihrer Hochschule einbinden können, wird am Praxisbeispiel des Projekts eScouts OER/MOOCs der Ruhr-Universität Bochum (RUB) gezeigt. Dazu wird das Projekt und die Arbeitsweise des studentischen eScouts-Teams, von der Recherche bis zur konkreten Umsetzung von Projektideen, vorgestellt. Eine der zentralen Massnahmen ist die von den eScouts entwickelte Plattform OpenRUB, auf der offen zugängliche bzw. frei lizenzierte Materialien aus Lehrveranstaltungen der RUB zur Verfügung gestellt werden. Hierfür wurde ein spezielles Kursformat adaptiert, das OpenCourseWare(OCW)-Format. Ferner wird dargelegt, wie Dozierende der RUB dafür gewonnen werden, Inhalte auf OpenRUB zur Verfügung zu stellen und wie sie sich OER mit Hilfe des eScouts-Teams aneignen können. Die einzelnen Projektschritte werden reflektiert und kritisch beleuchtet, um Schlüsse für die Fortführung des Projektes zu ziehen.
\end{abstract}

How students can help to open teaching processes in higher education

\begin{abstract}
As Open Educational Resources (OER) have come into focus in German higher education a few years ago it has also become an important goal of digitization to broadly offeropenness and freely licensed educational material in university teaching contexts. Students can be crucial multipliers in this process. The eScouts project at the Ruhr-Universität Bochum $(R \cup B)$ is a practice example that shows how student employees can help to implement the topic of Open Educational Resources (OER). The platform OpenRUB is an important part of the project. OpenRUB provides openly accessible and license-free teaching material. It is based on the adaptation of the OpenCourseWare (OCW) format. The article discusses how
\end{abstract}


professors and lecturers can be convinced to provide content on OpenRUB and how the eScouts tutor them in the usage of OER. The different steps in the process are evaluated to optimize the project.

\section{Einleitung}

In der Regel werden neue digitale Technologien und Anwendungsszenarien in der Hochschullehre von Lehrenden oder zentralen Einrichtungen eingeführt und weiterentwickelt. Dies gilt für eine Vielzahl von Technologien, die im Lehralltag genutzt werden: Lernplattformen, Veranstaltungsaufzeichnungen, speziell aufbereitete digitale Inhalte (z.B. Web based trainings, Animationen, Videos u.ä.) oder e-Prüfungen. In erster Linie entscheiden Lehrende, ob und welche Elemente Verwendung finden, die wiederum auch Studierenden zugutekommen sollen. Studierende als Adressaten von Hochschullehre haben hingegen weitaus weniger die Möglichkeit digitale Lehre mitzugestalten. Dabei wissen Studierende oft selbst am besten, was sie individuell zur Lernunterstützung benötigen (z.B. Zusatzmaterialien wie Texte, Übungsaufgaben, Lerngruppen, Visualisierungen etc.). Hinzu kommt, dass technologiebedingt eine autonome, selbstgesteuerte Handhabung des eigenen Lernprozesses verstärkt möglich geworden ist. Insbesondere im Hochschulstudium mit Blick auf die Forschungsorientierung ist dies ein wichtiger Bestandteil für das eigene erfolgreiche Studium. Es kann somit als erstrebenswert angesehen werden, dass Studierende an der Gestaltung digitaler Lehre partizipieren. Bei der Umsetzung des Projekts hatte das studentische eScouts-Team OER/MOOCs eigene Gestaltungsspielräume, war aber gleichwohl mit den Praktiken und Möglichkeiten der zentralen eLearning-Serviceeinrichtung der Ruhr-Universität Bochum (RUB) verknüpft. Aus dieser Konstellation heraus wurden Vorgehensweisen und Angebote entwickelt, um durch Studierende OER in der Hochschullehre zu verankern. Diese werden im folgenden Artikel dargestellt und reflektiert.

Für ein umfassendes Verständnis des Projekts und der Arbeitsweise werden zunächst die Rahmenbedingungen und Vorgehensweise des gesamten eScouts-Projekts genannt, das neben dem eScouts-Team OER/MOOCs aus zwei weiteren studentischen eScouts-Teams zu anderen Themen besteht. Es wird erklärt, wie und welche Ziele im Projektverlauf festgelegt wurden. In einem weiteren Schritt werden die Massnahmen der eScouts für die Umsetzung der Ziele dargelegt. Abschliessend wird das eScouts-Projekt OER/MOOCs reflektiert und bewertet. 


\section{Rahmenbedingungen des eScouts-Projekts}

Studierende erhalten im Projekt eScouts die Möglichkeit innerhalb der zentralen eLearning-Service-Einrichtung der RUB an der Gestaltung der digitalen Lehre mitzuwirken. Das Projekt eScouts OER/MOOCs soll es erlauben, sich mit Open Educational Resources (OER) und MOOCs in der Hochschullehre auseinander zu setzen, Anwendungsszenarien zu explorieren und Möglichkeiten zu finden, das Thema in der Hochschullehre zu platzieren. Dies impliziert auch die Lehre mithilfe von eLearning studierendenorientiert zu gestalten und ggf. Lehrveranstaltungen umzudenken und zu verändern. Zum besseren Verständnis des Projekts werden zunächst die Rahmenbedingungen dargelegt.

Das eScouts-Projekt wurde 2015 an der Ruhr-Universität Bochum vom Bereich eLearning (abgekürzt RUBeL) ins Leben gerufen und soll bis Anfang 2019 laufen. Finanziert wird das Projekt durch Qualitätsverbesserungsmittel für die Lehre der RUB. Als zentrale Service-Einrichtung berät und unterstützt RUBeL seit 2005 Lehrende beim Einsatz von e-Learning-Elementen in der Lehre. Dabei bezieht das RUBeL-Team seit zehn Jahren Studierende als beratende, unterstützende und ideengebende Akteurinnen und Akteure mit ein. Das RUBeL-Team entwickelt, begleitet und koordiniert Projekte, die gemeinsam mit Studierenden realisiert werden. Die studentischen Mitarbeiterinnen und Mitarbeiter können so auf eine vorhandene Struktur und Vernetzungen innerhalb der Hochschule zurückgreifen.

Die Einbeziehung von Studierenden ermöglicht eine realitätsnahe Einbindung von eLearning in die Hochschullehre, die bei der Unterstützung von Lehrenden auch berücksichtigt, dass Studierende Zielgruppe des Medienhandelns Lehrender sind und dass jeglicher Medieneinsatz Akzeptanz bei Studierenden finden muss. Vorteilhaft ist, dass Studierende neben ihrer Tätigkeit für das zentrale eLearning-Team der RUB auch Studierende im tatsächlichen Sinne sind, d.h., dass sie kontinuierlich Teil der alltäglichen Lehrendenpraxis sind und unmittelbaren Einblick in diese haben. Dadurch ist es möglich, konsequent die Perspektive der Studierenden bzw. deren Anforderungen an Lehrqualität und Ausrichtung des Lehrangebots mitzudenken. Ziel ist es, möglichst alle am Lehrprozess Beteiligten einzubeziehen. Darüber hinaus geht es auch darum, Erfahrungen zu sammeln, in neue Gebiete vorzustossen oder bestehende Praktiken zu verändern.

Die Besonderheiten und Charakteristika der Integration studentischer Mitarbeiterinnen und Mitarbeiter und Projekte finden sich auch im eScouts-Projekt wieder. Sie vereinen sich mit einem Teil des Aufgabenspektrums des RUBeL-Teams, bestimmte Trends und neue Entwicklungen im eLearning aufzugreifen und diese als Innovation mit Lehrenden zu erproben und gleichzeitig in den Lehralltag der RUB einzubinden (vgl. RUBeL 2009; Euler und Seufert 2004). Die eScouts sollen sich vornehmlich mit eLearning-Themen beschäftigen, die bisher an der RUB wenig bekannt sind oder kaum Eingang in die Lehre gefunden haben bzw. bei denen es keine etablierten 
Vorgehensweisen und Strukturen gibt. ${ }^{1}$ Teil des Projektes ist es, neue Formate, Angebote oder Aktionsformen zu entwickeln.

Im Rahmen des Projekts wurden insgesamt drei Teams gegründet. Ein Team besteht jeweils aus zwei Studierenden (auch eScouts genannt) und einer Ansprechpartnerin aus dem RUBeL-Team. Die Teams zeichnen sich durch Expertise im jeweiligen Thema aus und durch Offenheit und Kreativität dahingehend, für diese Themen Anwendungsformen in der Lehre zu finden. Folgende drei eScouts-Teams wurden aufgestellt:

- eScouts Inverted Classroom-Modell (ICM)

- eScouts Game Based Learning (GBL)

- eScouts Open Educational Resources (OER) \& Massive Open Online Courses (MOOCS)

Die Themen wurden gewählt, weil diese zum Entstehungszeitpunkt, so war zumindest der Eindruck und die Wahrnehmung seitens des RUBeL-Teams, verstärkt im Bildungstechnologie-Umfeld thematisiert und diskutiert wurden. Es sollte ausserdem grundsätzlich die Auseinandersetzung und Exploration neuer Entwicklungen stärker als bisher in den Blick genommen werden und auch zur Profilschärfung der zentralen eLearning-Dienste beitragen. Die Idee dabei war und ist weiterhin, dass solche Neuerungen verstärkt von Studierenden eingebracht werden sollten, im Sinne einer niedrigschwelligen und auch kostengünstigen Pilotierung. Ziel ist es, dass die eScouts-Teams Lehrende der RUB beim Einsatz von eLearning zu jeweils einem dieser Themenschwerpunkte beraten und unterstützen. Dabei werden die eScouts durch festangestellte Mitarbeiterinnen und Mitarbeiter des eLearning-Teams unterstützt. Diese fungieren als Projektleitung, Themenpatinnen und Themenpaten und direkte Ansprechpartnerinnen und Ansprechpartner der eScouts. Das Projektteam, das sich mit OER beschäftigt, wird in diesem Artikel vorgestellt, da es inhaltlich am besten zum Tagungsthema "Offenheit in Lehre und Forschung - Königsweg oder Sackgasse» passt. Das eScouts-Team OER/MOOCs hat allerdings im Projektverlauf eine Änderung des konkreten Themas erfahren: So wurde das ursprünglich zentrale Thema MOOCs mangels Interesses und Ressourcen an der RUB nicht weiter verfolgt. Stattdessen rückte das Thema "offene Bildungsmaterialien/OER» in den Fokus der eScouts-Arbeit, wodurch das Projekt eine thematisch breitere Ausrichtung bekommen hat.

Die eScouts-Projekte haben, unabhängig von den eLearning-Aktivitäten des RUBeL-Teams, aufgrund ihres Projektcharakters eine weitgehend eigenständige Stellung. Im Rahmen des Projekts bestehen grössere Freiheiten; eScouts können explorativer vorgehen. Im Bereich der eScouts-Themen bestand die Aufgabe daher auch

1 Damit wird dem kontinuierlichen Medienwandel und der Ubiquität digitaler Medien Rechnung getragen. Die Perspektive von E-Learning wird damit erweitert (vgl. Bachmann et. al 2009). 
darin, ein Beratungs-, Unterstützungs- und Umsetzungsformat erst zu entwickeln. Dies geschah dennoch nicht unabhängig von den Strukturen und Praktiken des RUBeL-Teams, die in erster Linie auf die Unterstützung von Lehrenden ausgerichtet sind. Bestehende Ansätze, Netzwerke und Strukturen des RUBeL-Teams wurden - wenn möglich und förderlich - mit den Aktivitäten des jeweiligen Projekts kombiniert.

Spezifisches eLearning-Vorwissen haben die eScouts vor allem durch die Teilnahme am Modul eTutoring erworben. In der vom RUBeL-Team konzipierten Lehrveranstaltung Modul eTutoring werden Studierende in technischer und didaktischer Hinsicht zu eTutoreninnen und eTutoren ausgebildet, um daraufhin mit Lehrenden während eines Semesters zusammen an der Umsetzung eines eLearning-Konzepts zu arbeiten (Thillosen und Hansen 2009; Henze und Cramer 2012)². Dabei haben die Studierenden erste Erfahrungen im Umgang mit eLearning und der Unterstützung von Lehrenden erhalten. Das Einbringen eigener Ideen und Ansätze ist hierbei bereits Bestandteil des eLearning-Einsatzes im Modul eTutoring. Neben der Erfahrung im Modul eTutoring kamen für die Tätigkeit und Qualifikation als eScout zusätzlich die eigene mediale Affinität und die Bereitschaft zur kontinuierlichen Weiterbildung hinzu. Wenngleich die eScouts im allgemeinen Sinn eine eLearning-Qualifikation durch die Teilnahme am Modul eTutoring erhalten haben, fehlte zu Beginn das konkrete Wissen zu OER bzw. MOOCs. Das für das Projekt relevante Fachwissen zu den Themen OER und MOOCs musste zunächst recherchiert und aufgebaut werden. Die eScouts erhielten Zeit und Gelegenheit sich durch Fachliteratur, Recherche und Unterstützung der Themenpatin die fachliche Basis anzueignen. Insbesondere zu Beginn des Projekts haben die eScouts die Diskussion und Auseinandersetzung mit OER im deutschsprachigen Raum rezipiert und aufgenommen, um einen eigenen Ansatz und eine Vorgehensweise für die RUB zu finden. Dies diente dazu, eine Orientierung für die praktische Umsetzung zu gewinnen und die daraus resultierende Praxis wiederum mithilfe der theoretischen Basis zu reflektieren und anzupassen. Eine darüber hinaus gehende Produktion von theoretischem Wissen war in dem Projekt nicht angelegt.

\section{Zielsetzungen des eScouts OER-Projekts}

Beobachtet haben die eScouts im Rahmen ihrer Recherche, dass die Themen MOOCs und OER mit dem Grundgedanken verbunden sind, Bildung für jeden zugänglich zu machen und damit die Universität zu öffnen (vgl. Deimann 2014). In der Regel werden damit weitergehende Ziele verfolgt, wie z.B. Bildungsgerechtigkeit (Deutsche UNESCO-Kommission 2013). Aus Studierendensicht deckt sich dies allerdings nicht mit der studentischen Erfahrung, denn in erster Linie sind an der RUB Lehr- und

2 Für das Modul eTutoring werden 5 oder 10 Credit Points vergeben. Ausserdem wird es als Berufsfeld-Praktikum für den Master of Education an der RUB anerkannt. 
Lerninhalte nicht öffentlich zugänglich, selbst wenn sie auf einer Lernplattform online gestellt werden. Diese Inhalte sind passwortgeschützt und nach dem Absolvieren der Lehrveranstaltung häufig für Studierende nicht mehr zugänglich. Mit offenen Inhalten kommen Studierende eher ausserhalb der Hochschule, z.B. auf YouTube oder mittels Wikipedia, in Berührung bzw. nutzen diese teilweise für das Studium. Gleichwohl ist aus Sicht der Studierenden ein offener und stetiger Zugriff sinnvoll, da bspw. zu einem späteren Zeitpunkt - nach dem Besuch einer Lehrveranstaltung erneut und zeit- sowie ortsunabhängig auf die Inhalte zugegriffen werden kann, z.B. um Seminararbeiten zu einem Veranstaltungsthema zu schreiben. Bei geschlossenen Formaten ist der Zugriff nicht mehr möglich oder wird alleinig durch den Lehrenden gesteuert.

Wenngleich es grundsätzlich als sinnvoll bzw. erstrebenswert erachtet wird, Bildungsmaterialien aus den genannten Gründen offen und frei zugänglich für jeden zur Verfügung zu stellen, ist es jedoch nicht ersichtlich, wie dieses Ziel aus der bestehenden Praxis heraus erreicht werden sollte. Im eScouts-Team stellte sich vordringlich die Frage, welche Auswirkungen die Nutzung von offenen Bildungsmaterialien auf die Lehr-/Lernpraxis haben kann, d.h. welche Vorteile sich für die Beteiligten an der Hochschule ergibt. Im Arbeitsverlauf wurde auch deutlich, dass die Zielsetzungen und die Bedeutung einer Öffnung für unterschiedliche Beteiligte (Studierende, Lehrende und ein externes Publikum) am Lehr-/Lernprozess, jeweils unterschiedlich sind bzw. sein können. Es kommt somit die Schwierigkeit in der Arbeit als eScout hinzu, auch für andere Rollen und Zielgruppen zu denken bzw. diese mitzudenken.

Neben möglichen Nachteilen, die sich vor allem aus der praktischen Umsetzung ergeben und später thematisiert werden, wurden Vorteile für Studierende in den folgenden Punkten gesehen:

- Lehr- und Studienmaterialien können in grösserem Masse zur Verfügung gestellt werden: Erfahrungsgemäss sind aus studentischer Sicht fachlich korrekte Lernmaterialien im Netz schwierig kostenfrei zu finden. Daher ist es grundsätzlich sinnvoll, dass auch universitäre Inhalte kostenlos und leicht zugänglich sind bzw. sein sollen.

- Neue Studierende können einen Einblick in die universitäre Lehre durch offen einsehbare Lehr- und Lernmaterialien bekommen.

- Diversität ${ }^{3}$, Individualität und Flexibilität von Organisations- und Lernprozessen (Deimann, Neumann, und Muuß-Merholz 2015) lassen sich fördern. OER können als Basis betrachtet werden, um Inhalte einfacher für spezifische Bedürfnisse und Zielgruppen aufzubereiten. Dies kann auch für Studierende insofern als vorteilhaft erachtet werden, als es individuelles Lernen ermöglicht.

3 Zum Begriff der Diversität: Im Leitbild der RUB ist die Wertschätzung von Vielfalt enthalten, die sich darin ausdrückt, dass Menschen aus unterschiedlichen Ländern, mit individuellen Lebensläufen, heterogenen Studienvoraussetzungen und vielfältigen Zielsetzungen und Erwartungen an der Universität zu finden sind (Ruhr-Universität Bochum 2017). 
- Studierende können ebenfalls die Möglichkeit bekommen Lerninhalte zu produzieren und zu veröffentlichen.

Demgegenüber liegen Vorteile für Lehrende in der Transparenz und Sichtbarkeit sowie der Möglichkeit die didaktische Qualität digitaler Lehre zu verbessern:

- Lehrinhalte und -methoden können sichtbar und transparent gemacht werden und als Anregung bzw. Grundlage für eigene Materialien und Aktivitäten dienen.

- OER kann allgemein als Baustein für die Verbesserung der didaktischen Qualität von Lehre begriffen werden (vgl. Deutsche UNESCO-Kommission 2015).

Aus den Vorteilen für Lehrende, so wird hier angenommen, ergeben sich auch Vorteile für Studierende. Diese können ebenfalls von einer Verbesserung der didaktischen Qualität profitieren. Mögliche Nachteile für Lehrende könnten darin liegen, dass durch eine Öffnung bzw. ein hohes Mass an Offenheit auch ein höherer Druck aufgebaut wird Qualität zu erzeugen und korrespondierend möglicherweise die Bereitschaft etwas auszuprobieren sinkt.

Im Laufe des Projekts haben sich die eScouts anhand von existierenden Beispielen jedoch davon überzeugen können, dass eine offene Lehr-/Lernpraxis zumindest möglich ist, z.B. in Form von OpenCourseWare, OER-Repositorien oder offenen Online-Kursen. Den potenziellen Vorteilen und dem herausgearbeiteten Mehrwert für Studierende durch offene Bildungsmaterialien/OER stehen jedoch nicht unerhebliche Umsetzungshürden auf dem Weg zu einer Öffnung von Lehr-/Lerninhalten gegenüber. Das Thema OER und offene Bildung ist zumindest in Deutschland erst seit wenigen Jahren bekannt geworden und es ist zu beobachten, dass sowohl bei Lehrenden als auch bei Studierenden in der Regel Unkenntnis bezüglich dieser Themen besteht. Auch die Relevanz für die eigene Lehr- und Studienpraxis scheint entsprechend nicht sehr hoch zu liegen. Dies lässt sich auf verschiedene Faktoren zurückführen, z.B. rechtliche und technische Rahmenbedingungen, nicht ausreichende Relevanz im Lehr- und Studienalltag, nicht verfügbare Zeit und Ressourcen bzw. auch die wenig ausgebildete Bereitschaft, Inhalte zu teilen (Deimann und Bastiaens 2010). Bei einigen dieser Punkte handelt es sich um Faktoren, die durch die bisherigen Massnahmen des eScouts-OER-Projekts nicht beeinflusst oder geändert werden können, z.B. die rechtlichen und technischen Rahmenbedingungen oder die verfügbare Zeit und vorhandenen Ressourcen. Im eScouts-OER-Projekt wird demgemäss versucht, Ansatzpunkte zu finden, bei denen OER in der Praxis relevant sein können und davon ausgehend Umsetzungsmöglichkeiten zu generieren.

Aus den Erfahrungen des eigenen Studiums konnten die eScouts feststellen, dass der Anteil an selbst erstellten Lehr/-Lernmedien bzw. Materialien noch gering ist. Jedoch können solche von Lehrenden selbst erstellten Inhalte, wie z.B. Skripte, Folien, 
Bilder, Videos, Aufgaben u.ä. als Ansatzpunkt für eine Umsetzung in Form offener Bildungsmaterialien/OER betrachtet werden.

Ausserdem erstellen Studierende mediale Artefakte im Rahmen von zu erbringenden Studienleistungen, die zwecks Bewertung zumeist nur Dozierenden zugänglich sind. In der Regel handelt es sich nicht um Medienproduktionen, die auch darüber hinaus von Interesse und Bedeutung sein können. Auch hier könnten Szenarien entwickelt werden, in denen Studierende Inhalte auf Basis von OER erstellen und diese veröffentlichen.

Um eine offene Lehr-/Lernpraxis bzw. eine Öffnung der Lehre einfach möglich zu machen, hat sich das eScouts-Team zunächst auf bereits vorhandene, potentiell für eine Öffnung in Frage kommende Inhalte konzentriert. Die Ausrichtung darauf, dass Studierende OER erstellen, wurde in Einzelfällen unterstützt, ist jedoch nicht primäres Ziel des Projekts. Dies liegt daran, dass eine Produktion von OER durch Studierende auch eine Änderung der didaktischen Konzeption von Lehrveranstaltungen nach sich ziehen müsste. Eine darauf abzielende Konzeptionierung scheint auch erst dann möglich zu sein, nachdem mehrere Erfahrungen und erste Schritte mit offenen Inhalten und OER gesammelt wurden. Teilweise wird aber in Beratungsgesprächen darauf verwiesen, dass diese Möglichkeit der OER-Produktion mit Einschluss von Studierenden besteht.

\section{Massnahmen im eScouts-Projekt OER/MOOCs}

Die Massnahmen setzten somit zunächst an der bestehenden digitalen Lehrpraxis an und versuchen aus dieser heraus Sichtbarkeit zu generieren. Daran gekoppelt sind die Entwicklung von Informationsangeboten und die Gewinnung von Lehrenden aus dem Kreis derjenigen, die bereits verstärkt eLearning-Angebote einsetzen und im Kontakt mit dem RUBeL-Team stehen. Der so gewählte, niedrigschwellige Ansatz zur Gewinnung von Lehrenden für konkrete OER-Umsetzungen orientiert sich am SAMR (Substitution, Augmentation, Modification, Redefinition)-Modell nach Puentedura (Puentedura 2016). Dieses Modell korrespondiert ebenfalls mit dem Ansatz eLearning Konzeptionen auf Veranstaltungsebene in ein Anreicherungs-, Integrations- und Virtualisierungskonzept zu unterteilen (Bachmann und Dittler 2004). Zum Beispiel stützt sich ein OCW-Kurs auf die Ersetzung (Substitution) bzw. Erweiterung (Augmentation) der bisherigen analogen und geschlossenen Lehre hin zu einer digitalen und offenen Lehrpraxis. Darauf aufbauend können zu einem späteren Zeitpunkt weitere Schritte folgen, die bis zu einer Änderung (Modification) oder sogar zu einer Transformation (Redefinition) der Lehrpraxis führen. 
Im Einzelnen handelt es sich um folgende Massnahmen, die von den eScouts festgelegt wurden:

- Bestehende Lehrpraxis: Moodle wurde als Ausgangspunkt zur Öffnung von Lehrinhalten ausgewählt. Die eScouts bieten die Umsetzung eines offenen Moodle-Kurses im OpenCourseWare-Format (OCW) an.

- Sichtbarkeit: Die öffentlich zugängliche Plattform OpenRUB wurde entwickelt, auf der offene Inhalte der RUB sichtbar werden.

- Zielgruppenansprache: Lehrende aus verschiedenen Projekten, in denen Inhalte selbst erstellt und aufbereitet worden sind, wurden konkret angesprochen mit dem Hinweis auf Beratung und auf ein Umsetzungsangebot.

- Wissens- und Sensibilisierungsangebote: Informationen zu OER und zur Öffnung von Lehrinhalten wurden erstellt.

Diese Massnahmen werden im Folgenden vertieft dargestellt.

Anknüpfung an die bestehende Lehrpraxis: Moodlekurse öffentlich zugänglich machen Zunächst wurde versucht ein Modell zu finden, wie bestehende Inhalte ohne grösseren Aufwand geöffnet und zugänglich gemacht werden können. Hierfür bietet sich aus Sicht des eScouts-Teams Moodle als niedrigschwelliger Ausgangspunkt dafür an, die Lehre zu öffnen und damit Lehrinhalte öffentlich zu präsentieren, da die meisten Lehrenden und Studierenden Moodle bereits für die Begleitung von Lehrveranstaltungen nutzen. Nachteilig für eine Öffnung ist, dass in Moodle-Kursen häufig urheberrechtlich geschütztes Material verwendet wird, personenbezogene Daten anfallen und organisatorische Informationen gegeben werden, die für eine breitere Öffentlichkeit nicht relevant sind. Daher hat das eScouts-Team ein OpenCourseWare-Format für die RUB entwickelt, welches an das OCW-Projekt des Massachusetts Institute of Technology ${ }^{4}$ angelehnt ist. Damit ist es möglich, Kursinhalte in einer einheitlichen Kursstruktur online zu veröffentlichen und nur die Inhalte, die für eine Veröffentlichung in Frage kommen, zu integrieren. OCW enthält somit vorrangig frei zugängliche und offen lizenzierte Publikationen von Bildungsmaterialien, die als Kurs organisiert sind: «Sie sind als Kurse angelegt und umfassen häufig Materialien zur Kursplanung sowie Bewertungsinstrumente und thematische Inhalte» (Deutsche UNESCO-Kommission 2013, 7; vgl. Sun, Wu, und Lee 2017). Im Unterschied zu OCW handelt es sich bei OER nicht nur um Kurse, sondern um alle Formen von Lernressourcen, weshalb OCW eine Untermenge von OER darstellt (ebd.). ${ }^{5}$

4 Massachusetts Institute of Technology (MIT) OpenCourseWare (OCW): https://ocw.mit.edu/index.htm.

5 "OCW does not mean online courses. The typical offering will consist of lecture notes, course outlines, reading lists, assignments, and similar course elements, as well as experiments, demonstrations, and students' work» (Sun, Wu, und Lee 2017). 
An der RUB stellen OCWs Moodle-Kurse zu vergangenen Lehrveranstaltungen dar. In einer einfachen Kursstruktur sind eine allgemeine Kursbeschreibung, der Lehrplan, Texte bzw. Literaturangaben enthalten. Darüber hinaus lassen sich auch Videos (wie bspw. selbst erstellte Vorlesungsaufzeichnungen) oder Übungs- und Klausuraufgaben einfügen. Für diese Inhalte liegen die Nutzungsrechte vor oder werden eingeholt. Vom eScouts-Team wird hierbei empfohlen die selbst erstellten Inhalte unter Creative Commons (CC)-Lizenzen zu veröffentlichen. ${ }^{6}$

Durch das OCW-Format kann somit zu einem bereits bestehenden Moodle-Kurs ein zusätzlicher, separater OCW-Kurs erstellt werden, der öffentlich zugänglich ist. Um den Aufwand für die Lehrenden bei der Umsetzung möglichst gering zu halten, bieten die eScouts die (technische) Erstellung eines solchen Kurses an.

Für Lehrende stellt das OCW-Format somit eine verhältnismässig einfache Umsetzungsform für die Öffnung von Kursinhalten dar. Während des Semesters arbeiten Lehrende und Studierende weiterhin mit ihren geschlossenen Moodle-Kursen. Das OCW-Format besteht somit unabhängig vom Originalkurs. Die OCW-Kurse haben einerseits zum Ziel die Lehre der Dozierenden zu präsentieren und andererseits Studieninteressierten die Möglichkeit, Einblick in spezifische Veranstaltungen der RUB zu geben, um z.B. die Studienwahl zu unterstützen, sich zu orientieren oder Interessen zu wecken. Ein OCW-Kurs bietet daher sowohl für Lehrende als auch für Studierende Vorteile. Diese Möglichkeit der Online-Präsentation und -Vorstellung von Lehrinhalten gab es an der RUB für Studierende bislang nicht. Die einheitliche OCW-Kursstruktur schafft Übersichtlichkeit und kann dadurch die Nutzung digitaler Lehrinhalte erleichtern, was häufig innerhalb der Moodle-Kurse nicht gegeben ist. Studierende haben ausserdem damit grundsätzlich die Möglichkeit unabhängig von Kursen auf Studien- und Lerninhalte zuzugreifen.

\section{Öffentliche Sichtbarkeit von Lehrinhalten: OpenRUB-Plattform}

Um die offenen Inhalte auffindbar zu machen, wurde es als notwendig erachtet, auch einen zentralen Ort an der RUB zu haben, an dem diese zu finden sind. Hierzu wurde die Plattform OpenRUB ${ }^{7}$ entwickelt, um offene Inhalte zu sammeln und zugänglich zu machen.

Die Inhalte sind frei zugängliche und offene Lehr-/Lernmaterialien, die in der Regel im Rahmen von Lehrveranstaltungen entstanden sind, z.B. OCW-Kurse. Jedoch haben die eScouts auf der Plattform OpenRUB neben OCW-Kursen weitere Formate integriert: Informationskurse, vollständige Selbstlernkurse oder Vorlesungsaufzeichnungen. Diese Formate können jederzeit nach Bedarf angepasst und erweitert

6 Hinter Creative Commons steht eine Non-Profit-Organisation, die Urheber/innen vorgefertigte Lizenzverträge zur Nutzung kreativer Materialien (u.a. Text, Bild, Ton) durch Dritte anbietet. Mehr unter: https:// creativecommons.org/ (Creative Commons 2018).

7 OpenRUB: https://open.ruhr-uni-bochum.de. 
werden, denn die genannten Typen sind nicht als abgeschlossen zu verstehen. Auch einzelne Folien, Skripte, Bilder, Videos oder Daten, die im Lehr-/Lernkontext entstanden sind, und für Aussenstehende von Interesse sein können, lassen sich hinzufügen. Die Arbeit an der Plattform versteht sich - wie ein grosser Teil der eScouts-Tätigkeit - als prozessorientierte Arbeit. Dementsprechend werden Aufbau und Funktionen der Plattform stetig vom eScouts-Team reflektiert, weiterentwickelt und ausgebaut. Auf der Grundlage von neuen Erfahrungen und Rückmeldungen von Lehrenden und Studierenden wurde so bereits im Mai 2018 eine aktualisierte Version der Plattform veröffentlicht. Angestrebt wird, dass auf OpenRUB alle frei und offen verfügbaren Lehr- und Lernmaterialien, die von Angehörigen der RUB erstellt wurden, gesammelt und kategorisiert werden. Ziel ist es, damit langfristig ein «OER-Repositorium» (vgl. Deutsche UNESCO-Kommission 2015) der RUB aufzubauen. Inhalte, die unter freier Lizenz stehen, werden auf OpenRUB auf einer Übersichtsseite durch ein OER-Logo und einer (CC) Lizenzangabe gekennzeichnet. Dies ermöglicht den Nutzenden einen einfachen Überblick über OER-Materialien.

Im Verständnis des eScouts-OER-Projektes soll es möglich sein, Inhalte nur mit der Option des kostenlosen Zugangs zu veröffentlichen, ohne die Verwendung einer offenen Lizenz. Die Gründe dafür sind, zunächst Lehrende mit der Offenheit von Inhalten vertraut zu machen, diese zu sensibilisieren und einen niedrigschwelligen Ansatz für die Öffnung von Lehrinhalten anzubieten.

Zurzeit werden über OpenRUB mehr als 50 frei zugängliche Lehr- und Lernmaterialien bereitgestellt. Darunter sind OCW-Kurse, Selbstlernkurse, Demokurse, Informationsangebote zu lehr- oder studienbezogenen Themen, Vorlesungsaufzeichnungen und einzelne Lehr-/Lernmaterialien unterschiedlicher Granularität (von einzelnen Folien bis zu Webseiten). Durch eine Suchfunktion und Sortierung der Inhalte in Fachbereiche können Nutzerinnen und Nutzer auf für sie relevante Materialien zugreifen. Einen Teil der Inhalte (fünf Moodle-Kurse) haben die drei eScouts-Teams selbst entwickelt und unter CC lizenziert. Teilweise wurden Kurse von Lehrenden in Kooperation mit den eScouts-Teams umgesetzt. Denkbar ist, dass OpenRUB verstärkt auch auf die Bedarfe und Anforderungen von Studierenden ausgerichtet wird. Es könnten z.B. vermehrt Inhalte zugänglich gemacht werden, die von übergreifendem Interesse und Belang sind, z.B. Materialien zum wissenschaftlichen Arbeiten. Es ist auch in Betracht zu ziehen, dass Studierende zukünftig Materialien auf OpenRUB zur Verfügung stellen können.

Bei der Auswahl und Durchsicht der Inhalte, die auf OpenRUB veröffentlicht werden, spielen die eScouts eine massgebliche Rolle. Die eScouts beraten und geben ausserdem Tipps im Hinblick auf Design, Inhalt und didaktischen Aufbau. Diese Beratung kann für die Dozierenden einen Mehrwert darstellen, da sie von Studierenden, die auch technisches Wissen über Veränderungsmöglichkeiten haben, ein Feedback zum Moodle-Kurs einholen können. 


\section{Zielgruppenansprache: Kontaktaufnahme zu Lehrenden}

Um Lehrende zu gewinnen, die ihre Lehre öffnen möchten, hat das eScouts-Team es als vorteilhaft erachtet, Personen anzusprechen, die sich bereits intensiver mit der Aufbereitung von eLearning-Inhalten beschäftigen, d.h. eLearning-Werkzeuge und -Inhalte einsetzen, die über das Bereitstellen von Dokumenten hinausgehen.

Damit wären zumindest Inhalte vorhanden, die eine Öffnung interessant erscheinen lassen würden. Dazu zählen in erster Linie die Kontaktaufnahme zu:

- Gewinnerinnen und Gewinnern des RUB-internen eLearning-Wettbewerbs $5 \times 5000^{8}$,

- Teilnehmenden am Modul eTutoring,

- Dozierenden, deren Kurse ein eLabel ${ }^{9}$ tragen,

- Dozierenden, die ihre Veranstaltung über Ton oder Video für Studierende aufzeichnen lassen,

- Dozierenden, die vom RUBeL-Team bereits umfänglicher beraten werden.

Das eScouts-Team trat und tritt mit Lehrenden auf unterschiedlichen Wegen in Kontakt: über E-Mails, Telefongespräche und persönliche Beratungen. Insgesamt wurden bereits über 100 persönliche Gespräche, Telefonate und E-Mails mit Dozierenden geführt und geschrieben. Dadurch konnten zum Teil Personen gewonnen werden, die zu einer Öffnung ihrer Lehre bereit waren. Diese Materialien lassen sich auf OpenRUB finden. Allerdings wurde hierbei besonders deutlich, dass die Öffnung der Lehre oder die Verwendung von OER im Allgemeinen von Seiten der Lehrenden nur geringfügig nachgefragt ist. Dies liegt zum Teil auch daran, dass die Inhalte zu einem grossen Teil urheberrechtlich geschützt sind, z.B. im Fall von Vorlesungsaufzeichnungen, und nicht ohne Weiteres geöffnet werden können.

\section{Erstellung von Informations- und Sensibilisierungsangeboten}

Da der Bekanntheitsgrad von OpenRUB/OER und das Wissen zu OER bei Lehrenden und Studierenden bisher wenig ausgeprägt ist, haben die eScouts Informationsangebote zu offenen Bildungsmaterialien/OER entwickelt.

Das studentische Team musste sich selbst in die OER-Thematik einarbeiten und konnte sich daher in die Lage zurückversetzen, welche Aspekte besonders schwierig zu verstehen und besonders wichtig sind, wie z.B. das Wissen über das Urheberrecht und die Lizenzierungsmöglichkeiten. Im Studium werden Studierende mit fehlerhaften bzw. mangelhaften Lizenzangaben auf Präsentationsfolien (von Studierenden und Dozierenden) konfrontiert, wodurch eine fehlerbehaftete Arbeitskultur vorgelebt

8 Der 5x5000-Wettbewerb findet an der RUB jedes Semester statt, bei dem fünf eLearning-Projekte mit je $5000 €$ gefördert werden. Die Gewinner/innen werden von einer studentischen Jury ausgesucht.

9 Das eLabel ist das Qualitätssiegel der RUB für gutes eLearning. Damit werden begleitenden Moodle-Kurse nach einem Kriterienkatalog ausgezeichnet. 
wird. Daher ist es dem eScouts-Team wichtig Informationsmaterial zu diesen Themen bereitzustellen. Die Informationsangebote können freiwillig und unverbindlich genutzt werden und sind ebenfalls auf OpenRUB zu finden. Zum Kompetenzaufbau von OER-Interessierten entwickelten die eScouts z.B. eine Online-Selbstlerneinheit in Moodle zu OER und CC-Lizenzen. ${ }^{10}$ Schwerpunkte sind hierbei: Was sind OER? Was sind CC-Lizenzen und wie funktionieren diese? Wie können OER für die eigene Lehre gefunden werden? Wie können die eigenen Materialien als OER und unter CC-Lizenzen veröffentlicht werden?

Auch für dieses Online-Angebot ist es schwierig Nutzerinnen und Nutzer zu finden. Um dabei erfolgreicher zu sein, wäre es beispielsweise günstig, dies in Angebote zur Vermittlung von Methoden wissenschaftlichen Arbeitens zu integrieren. Eine Gelegenheit innerhalb einer Lehrveranstaltung Basiswissen zu OER zu vermitteln, ist im Rahmen der Lehrveranstaltung des Moduls eTutoring gegeben. Dort ist das eScoutsTeam zu Anfang und zum Ende der Lehrveranstaltung daran beteiligt Basiswissen zu OER zu vermitteln und zu einer Öffnung von Lehrmaterialien zu motivieren. Auf diese Weise geben die eScouts ihren studentischen Kommilitonen Anregungen und Informationen, damit diese im Rahmen ihrer Tätigkeit als eTutorinnen und eTutoren bei der Aufbereitung von Lehrmaterialien OER verwenden. Dies ist auch, so ist zu beobachten, teilweise geschehen. Eine systematische Überprüfung wurde bisher allerdings nicht vorgenommen.

\section{Bewertung und Fortführung des Projekts}

Das eScouts-OER-Projekt hat dem Thema offene Bildungsmaterialien/OER an der Ruhr-Universität Bochum durch die Etablierung der Plattform OpenRUB erste Sichtbarkeit verschafft und versucht mit Informationsangeboten auf die Thematik aufmerksam zu machen. Auf dem Weg zur Realisierung der anfänglichen Idee, dass die Nutzung von OER in der universitären Lehre an der RUB selbstverständlich(er) wird und dass offene Bildungsmaterialien in vielen Lehrveranstaltungen zum Einsatz kommen, sind damit erste Schritte eingeleitet worden. Auf der Plattform OpenRUB sind demgemäss einige offene Inhalte zu finden. Eine offene Plattform kann in diesem Kontext als ein Baustein gelten, diese Aktivitäten sichtbar zu machen. Darüber hinaus gibt es zum Teil davon ausgehend einzelne Beratungs- und Realisierungsanfragen im Kontext von Lehrveranstaltungen und Lehrprojekten. Hauptsächlich wurde im eScouts-OER-Projekt in einem ersten Schritt versucht aus der bestehenden Lehr-/Lernpraxis heraus möglichst niedrigschwellig Inhalte sichtbar zu machen, d.h. zu öffnen und dafür einen Mehrwert für Dozierende, Studierende und auch Studieninteressierte zu formulieren. Dabei konzentrierten sich die eScouts in einem ersten Anlauf auf die Veranstaltungen/Lehrenden, die in gewissem Masse digitale Inhalte

10 Einführung OER: https://moodle.ruhr-uni-bochum.de/m/course/view.php?id=8732. 
aufbereiten und erstellen, d.h. mehr anbieten als die Verfügbarmachung von Literatur, z.B. Tests, Skripte, Videos oder Lerneinheiten u.ä. Insbesondere wurde versucht einen Nutzen und Mehrwert für Studierende zu berücksichtigen. Dieser Perspektive kam zugute, dass das eScouts-Projektteam aus Studierenden gebildet wurde.

Anschliessend an das so genannte «transformative Potential» von OER (Deutsche UNESCO-Kommission 2013, 7) ist im eScouts-OER Projekt die Einsicht gewachsen, dass es sinnvoll und erstrebenswert ist, OER und offene Angebote im Hinblick auf eine Veränderung und Verbesserung der Lehr- und Lernpraxis unmittelbar in die Lehre zu integrieren. Unsicherheiten bezüglich des Urheberrechts und den CC-Lizenzierungsmöglichkeiten müssten aus eScouts-Sicht beseitigt werden, um das Thema OER zu stärken und in diesem Zusammenhang Konzepte zu realisieren, in denen Studierende eine aktivere Rolle erhalten, z.B. indem OER unmittelbar als Lehrbaustein in die Lehre integriert werden. Bestandteil eines solchen Lehrangebotes könnte es sein, dass Studierende die Möglichkeit erhalten Inhalte als OER, in verschiedenen medialen Ausprägungen, zu produzieren. Dies kann zum einen dann geschehen, wenn sich dies aus fachlicher Sicht nahelegt, aber auch als Bestandteil von Medienkompetenz, als Fähigkeit Inhalte so zu erstellen, dass sie nachnutzbar und offen sind. Eine weitere Möglichkeit bestünde darin, dass Gelegenheit gegeben wird, mediale Projekte zu realisieren.

Abschliessend kann zusammengefasst werden, dass innerhalb des Projekts eScouts erste niedrigschwellige Schritte umgesetzt wurden, um durch Studierende das Thema OER Lehrenden näher zu bringen. Dies resultiert aus den bereits vorhandenen Strukturen des RUBeL-Teams und den Freiheiten, die im Projekt gegeben sind, sodass die im Projekt entstandenen Ideen ohne grössere Hürden umgesetzt werden konnten. Das Projekt zeigt auch, dass ein Bottom-Up-Verfahren in einer von Hierarchien durchzogenen Organisation wie der Hochschule funktionieren kann. Es muss allerdings auch gesehen werden, dass ein Bottom-Up-Verfahren an seine Grenzen stösst, wenn es um die flächendeckende Akzeptanz und Implementierung von OER geht. Es hat sich im eScouts-Projekt die Meinung gefestigt, dass - um eine strukturelle Veränderung der Lehrpraxis zu erzielen - ebenfalls eine Verankerung «von oben» nach dem Top-Down-Prinzip erfolgen sollte (vgl. Orr et al. 2017). Dies könnte durch eine hochschulweite OER-Policy geschehen. Eine solche OER-Policy kann eine hochschulweite Übereinkunft darstellen, dass OER ein wichtiger Teil der Lehr- und Lernpraxis sind, analog zu Open Access-Fördermassnahmen. Dazu gehört es begleitend die Relevanz im Lehralltag und die Bereitschaft Inhalte zu teilen über das eScoutsOER-Projekt hinaus kontinuierlich und über einen längeren Zeitraum zu unterstützen und mit einem sichtbaren organisationalen Stellenwert zu versehen. 


\section{Literatur}

Bachmann, Gudrun, Antonia Bertschinger, und Jan Miluska. 2009. «E-Learning ade - tut Scheiden weh?». In E-Learning 2009. Lernen im digitalen Zeitalter, hrsg. v. Nicolaus Apostolopoulos, Harriet Hoffmann, Veronika Mansmann und Andreas Schwill, 118-128. München u.a.: Waxmann.

Bachmann, Gudrun, und Martina Dittler. 2004. «Integration von E-Learning in die Hochschule: Umsetzung einer gesamtuniversitären Strategie an der Universität Basel». In E-LearningStrategien und E-Learning-Kompetenzen an Hochschulen, hrsg. v. Claudia Bremer und Kerstin E. Kohl, 47-61. Bielefeld: Bertelsmann.

Creative Commons. 2018. «Creative Commons Deutschland mehr Möglichkeiten». https://creativecommons.org/.

Deimann, Markus. 2014. «Open Education and Bildung: Ideas, Assumptions, and Their Vigour to Transform Higher Education.». MedienPädagogik (24): 94-113. https://doi.org/10.21240/ mpaed/24/2014.09.15.X.

Deimann, Markus, Jan Neumann, und Jöran Muuß-Merholz. 2015. Whitepaper Open Educational Resources (OER) an Hochschulen in Deutschland - Bestandsaufnahme und Potenziale 2015, hrsg. v. Transferstelle für OER. https://open-educational-resources.de/wp-content/ uploads/Whitepaper-OER-Hochschule-2015.pdf.

Deimann, Markus, und Theo Bastiaens. 2010. «Potenziale und Hemmnisse freier digitaler Bildungsressourcen - eine Delphi-Studie.». In Zeitschrift für E-learning, Nr. 5, 7-18. Wien: Studienverlag.

Deutsche UNESCO-Kommission. 2013. Was sind Open Educational Resources? Und andere häufig gestellte Fragen zu OER. Bonn. https://www.unesco.de/sites/default/files/2018-04/ Was_sind_OER__cc.pdf.

Deutsche UNESCO-Kommission. 2015. Leitfaden zu Open Educational Resources in der Hochschulbildung. Empfehlungen für Politik, Hochschulen, Lehrende und Studierende. Bonn. https://www.unesco.de/fileadmin/medien/Dokumente/Bildung/DUK_Leitfaden_OER_in_ der_Hochschulbildung_2015_barrierefrei.pdf.

Euler, Dieter, und Sabine Seufert. 2004. «Von der Pionierphase zur nachhaltigen Implementierung - Facetten und Zusammenhänge einer pädagogischen Innovation». In E-Learning in Hochschulen und Bildungszentren, hrsg. v. Dieter Euler und Sabine Seufert, 1-24. München u.a.: Oldenbourg.

Henze, Simone, und Michael Cramer. 2012. "Universitas: Lehrende lernen von Studierenden im Rahmen des Moduls eTutoring». In Grundfragen Multimedialen Lehrens und Lernens. Von der Innovation zur Nach haltigkeit: Tagungsband GML ${ }^{2} 2012$, hrsg. v. Nicolas Apostolopoulos, Wolfgang Coy, Ulrike Mußmann und Andreas Schwill, 217-234. Münster: Waxmann Verlag.

Orr, Dominic, Jan Neumann, und Jöran Muuß-Merholz. 2017. German OER Practices and Policy - from Bottom-up to Top-down Initiatives. Moskau: UNESCO Institute for Information Technologies in Education. https://iite.unesco.org/pics/publications/en/files/3214746.pdf.

Puentedura, Ruben. 2006. «Transformation, Technology, and Education». http://www.hippasus.com/resources/tte/. 
RUBeL. 2009. «Leitbild eLearning». https://www.rubel.rub.de/content/leitbild-elearning.

Ruhr-Universität Bochum. 2017. «Vielfalt und Chancengleichheit an der RUB». http://www. ruhr-uni-bochum.de/universitaet/profil/vielfalt-und-chancengleichheit/index.htm.

Sun, Jerry Chih-Yuan, Yu-Ting Wu, und Wei-I Lee. 2017. "The effect of the flipped classroom approach to OpenCourseWare instruction on students' self-regulation». British Journal of Educational Technology 48(3): 713-729. https://doi.org/10.1111/bjet.12444.

Thillosen, Anne, und Holger Hansen. 2009. «Technik und Didaktik im E-Learning: Wer muss was können? Ein Plädoyer für verteilte Medienkompetenz in Hochschulen». In E-Learning: Eine Zwischenbilanz. Kritischer Rückblick als Basis eines Aufbruchs, hrsg. v. Ullrich Dittler, Jakob Krameritsch, Nicolae Nistor, Christine Schwarz und Anne Thillosen, 133-148. Münster: Waxmann Verlag. 